\title{
LIE METABELIAN SKEW ELEMENTS IN GROUP RINGS
}

\author{
GREGORY T. LEE \\ Department of Mathematical Sciences, Lakehead University, \\ Thunder Bay, Ontario P7B 5E1, Canada \\ e-mail: glee@lakeheadu.ca \\ and ERNESTO SPINELLI \\ Dipartimento di Matematica 'G. Castelnuovo', Università degli Studi di Roma \\ 'La Sapienza', P. le Aldo Moro n. 5, Rome 00185, Italy \\ e-mail:spinelli@mat.uniromal.it
}

(Received 14 July 2012; accepted 22 January 2013; first published online 13 August 2013)

\begin{abstract}
Let $F$ be a field of characteristic $p \neq 2$ and $G$ a group without 2elements having an involution $*$. Extend the involution linearly to the group $\operatorname{ring} F G$, and let $(F G)^{-}$denote the set of skew elements with respect to $*$. In this paper, we show that if $G$ is finite and $(F G)^{-}$is Lie metabelian, then $G$ is nilpotent. Based on this result, we deduce that if $G$ is torsion, $p>7$ and $(F G)^{-}$is Lie metabelian, then $G$ must be abelian. Exceptions are constructed for smaller values of $p$.
\end{abstract}

2010 Mathematics Subject Classification. 16S34, 16R50.

1. Introduction. Let $F G$ be the group ring of a group $G$ over a field $F$ of characteristic $p \neq 2$. If $G$ has an involution $*$, then we extend it linearly to obtain an involution of $F G$, also denoted by $*$. We write $(F G)^{-}$for the set of all skew elements of $F G$, that is, those elements $\alpha$ satisfying $\alpha^{*}=-\alpha$. It is easy to see that $(F G)^{-}$is the Lie sub-algebra of $F G$ consisting of all linear combinations of terms of the form $g-g^{*}$, with $g \in G$. A general problem in group rings is to decide the extent to which the structure of $(F G)^{-}$determines the structure of $F G$. If $g^{*}=g^{-1}$ for all $g \in G$, then the induced involution is called the classical involution. During the last two decades, a considerable amount of attention has been devoted to determining if Lie properties satisfied by $(F G)^{-}$are also satisfied by $F G$. Giambruno and Sehgal in [8] showed that if $G$ has no 2-elements and $(F G)^{-}$is Lie nilpotent, so is $F G$. Lee [9] proved the analogous result for the bounded Lie Engel property. Lie solvability was considered by Lee et al. [11] with suitable restrictions upon $G$. More recently, a great deal of work has appeared considering involutions other than the classical one. For example, the conditions under which the skew elements commute were determined in Broche et al. [4]. Subsequently, Giambruno et al. [7] determined the torsion groups without 2-elements such that $(F G)^{-}$is Lie nilpotent, and Catino et al. handled the bounded Lie Engel property in [5]. We note that the situation with the skew elements is more involved than the corresponding problem for the symmetric elements. Indeed, the absence of 2-elements was not sufficient to force $F G$ to have the same property in these papers; there were exceptional cases.

This type of work has inspired similar investigations in other specific classes of algebras with involution. For instance, Siciliano [17] has characterized restricted Lie 
algebras $L$ over a field of characteristic $p>2$ such that the set of skew elements of the restricted enveloping algebra $u(L)$ with respect to the principal involution is Lie solvable, Lie nilpotent or bounded Lie Engel. In the same paper, the Lie structure of the set of skew elements of the ordinary enveloping algebra $U(L)$ is also discussed.

On any ring $R$, let $[x, y]=x y-y x$. We say that a subset $S$ of $R$ is Lie metabelian if $\left[\left[s_{1}, s_{2}\right],\left[s_{3}, s_{4}\right]\right]=0$ for all $s_{i} \in S$. Our purpose here is to examine the conditions under which $(F G)^{-}$is Lie metabelian, where $G$ is a torsion group without 2-elements having an arbitrary involution. The main part of the work involves proving the following.

THEOREM 1. Let $F$ be a field of characteristic different from 2 and $G$ a finite group of odd order having an involution. Extend the involution linearly to $F G$. If $(F G)^{-}$is Lie metabelian, then $G$ is nilpotent.

Levin and Rosenberger in [14] showed that if $G$ is a finite group with no 2- or 3 -elements, and $F G$ has the classical involution, then $G$ is abelian whenever $(F G)^{-}$is Lie metabelian. Using Theorem 1, we are able to extend their result. First, provided $p>3$, we can remove the restriction on 3-elements. Second, if $p>7$, we can allow a general involution on $G$.

THEOREM 2. Let $F$ be a field of characteristic $p \notin\{2,3\}$ and $G$ a torsion group with involution, having no elements of order 2. Extend the involution linearly to $F G$, and let $(F G)^{-}$be Lie metabelian. Then

1. if the involution on $F G$ is classical, then $G$ is abelian, and

2. if $p \notin\{5,7\}$ as well, then $G$ must be abelian regardless of the involution.

As we shall see, the restrictions upon the characteristic in Theorem 2 are necessary. When $p \in\{5,7\}$, the fact that $(F G)^{-}$is Lie metabelian does not imply that $F G$ is also Lie metabelian for an arbitrary involution.

2. Preliminaries. Let us gather some necessary results. Let $F$ be a field and $R$ an $F$-algebra. If $S$ is any subset of $R$, let $\delta^{[0]}(S)$ be the subspace of $R$ spanned by $S$, and for all positive integers $i$, let $\delta^{[i]}(S)$ be the subspace of $R$ spanned by the terms $\left[s_{1}, s_{2}\right]$, with $s_{i} \in \delta^{[i-1]}(S)$. We say that $S$ is Lie solvable if there exists an $i$ such that $\delta^{[i]}(S)=0$. The smallest such $i$ is called the Lie derived length of $S$. (Clearly, $S$ is Lie metabelian precisely when its Lie derived length is at most 2.)

In Catino et al. [6], the following two results were stated for the set of symmetric elements, $(F G)^{+}=\left\{\alpha \in F G: \alpha^{*}=\alpha\right\}$. (These were originally proved in [11, 12] for the classical involution, but the involution did not matter for the relevant portion of the proof.) Since $\left[(F G)^{+},(F G)^{+}\right] \subseteq(F G)^{-}$, it is clear that if $(F G)^{-}$is Lie solvable, so is $(F G)^{+}$. Thus, we have the following.

LEMMA 1. Let $G$ be a finite group of odd order having an involution *. Let $F$ be a field of characteristic different from 2 , and extend the involution linearly to $F G$. Suppose that $F G$ is semi-simple. If $(F G)^{-}$is Lie solvable, then $G$ is abelian.

LEMMA 2. Let $G$ be a finite group of odd order having an involution $*$, and let $F$ be a field of characteristic $p>2$. Extend the involution linearly to $F G$. If $(F G)^{-}$is Lie solvable, then the p-elements of $G$ form a (normal) subgroup $P$, and $G / P$ is abelian.

Not too much is known about Lie derived lengths in general. However, the next two results will be useful. We write $G^{\prime}$ for the derived subgroup of $G$. First, for the classical involution there is the following. 
LEMMA 3. Let $F$ be a field of characteristic $p>2$ and $G$ a nilpotent group with $G^{\prime} a$ cyclic p-group. With respect to the classical involution, the Lie derived length of $(F G)^{-}$is $\left\lceil\log _{2}\left(\left|G^{\prime}\right|+1\right)\right\rceil$.

Proof. See Balogh and Juhász [3].

On the other hand, a lower bound for the Lie derived length of $(F G)^{+}$is provided for a non-abelian $p$-group $G$ with an arbitrary involution.

LEMMA 4. Let $F$ be a field of characteristic $p>2$ and $G$ a finite non-abelian p-group with involution. Let $F G$ have the induced involution. Then the Lie derived length of $(F G)^{+}$ is at least $\left\lceil\log _{2}(p+1)\right\rceil$.

Proof. See Balogh [2].

We also require the following two group-theoretic lemmas.

LEMMA 5. Let $A$ be an abelian torsion group with no 2-elements, having an involution *. Then $A=A_{1} \times A_{2}$, where $A_{1}$ is the set of symmetric elements of $A$ and $A_{2}=\{a \in A$ : $\left.a^{*}=a^{-1}\right\}$.

Proof. See [7, Lemma 2.7].

We will use the $A_{1}$ and $A_{2}$ notations without further mention.

LEMMA 6. Let $G$ be a torsion group without 2-elements having an involution $*$ such that $G$ has a *-invariant abelian normal subgroup $A$. Then

1. if $x \in G \backslash A$ satisfies $x^{*} \in x^{-1} A$, then there exists $e \in A_{1}$ such that $(x e)^{*}=(x e)^{-1}$, and

2. if $A$ is finite, $x \in G$ satisfies $x^{*} \in x A$, and $o(x)$ is relatively prime to $|A|$, then there exists $e \in A_{1}$ such that $(x e)^{*}=x e$.

Proof. The first part is [7, Lemma 2.8]. To obtain the second part, note that $A \rtimes\langle x\rangle$ is $*$-invariant, and apply Remark 3.1 and Lemma 2.8 in [7].

Recall that if $R$ is an $F$-algebra, then the subset $S$ of $R$ is said to satisfy a polynomial identity if there exists a non-zero polynomial $f\left(x_{1}, \ldots, x_{n}\right)$ in the free algebra on non-commuting indeterminates $F\left\{x_{1}, x_{2}, \ldots\right\}$ such that $f\left(s_{1}, \ldots, s_{n}\right)=0$ for all $s_{i} \in S$. Obviously, when $(F G)^{-}$is Lie metabelian, it satisfies a polynomial identity. This allows us to make use of the following classical result due to Amitsur [1].

Lemma 7. Let $F$ be a field and $R$ an $F$-algebra with involution. If $R^{-}$satisfies a polynomial identity, so does $R$.

Proof. See [1].

Group rings satisfying a polynomial identity were classified by Isaacs and Passman. Recall that $G$ is $p$-abelian if $G^{\prime}$ is a finite $p$-group, and that 0 -abelian means abelian.

LeMmA 8. Let $F$ be a field of characteristic $p \geq 0$ and $G$ a group. Then FG satisfies a polynomial identity if and only if $G$ has a p-abelian subgroup of finite index.

Proof. See [15, Corollaries 5.3.8 and 5.3.10].

Combining the last two lemmas, we observe that we can reduce our considerations from torsion groups to locally finite groups. 
3. Proofs of the main results. In any group, let us write $a^{b}=b a b^{-1}$ and $(a, b)=$ $a^{-1} b^{-1} a b$.

Proof of Theorem 1. If char $F=0$, then Lemma 1 does the job, so let char $F=$ $p>2$. Suppose that the theorem fails, and let $G$ be a counterexample of the smallest order. If the centre, $\zeta$, is nontrivial then, since $(F(G / \zeta))^{-}$is still Lie metabelian, we see that $G / \zeta$ is nilpotent, and so is $G$. Thus, $G$ is centreless. By Lemma 2 , the $p$-elements of $G$ form a normal subgroup $P$, and $G / P$ is abelian. If $P$ is not abelian, then noting that $\left(F\left(G / P^{\prime}\right)\right)^{-}$is Lie metabelian, we see that $G / P^{\prime}$ is nilpotent. By Hall's criterion (see [16, 5.2.10]), $G$ is nilpotent. Thus, $P$ is abelian.

By the Schur-Zassenhaus theorem, $G=P \rtimes X$, for some $p^{\prime}$-subgroup $X$. It suffices to show that $(a, x)=1$ for all $a \in P, x \in X$. By Lemma 5, $P=P_{1} \times P_{2}$ and $G / P=(G / P)_{1} \times(G / P)_{2}$. Write $a=a_{1} a_{2}$ with $a_{i} \in P_{i}$, and $P x=\left(P x_{1}\right)\left(P x_{2}\right)$, with $P x_{i} \in(G / P)_{i}$. We are done if we can show that each $\left(a_{i}, x_{j}\right)=1$. By Lemma 6 , there exist $e_{j} \in P_{1}$ such that $\left(x_{1} e_{1}\right)^{*}=x_{1} e_{1}$ and $\left(x_{2} e_{2}\right)^{*}=\left(x_{2} e_{2}\right)^{-1}$. Now each $a_{i}$ commutes with $x_{j}$ if and only if it commutes with $x_{j} e_{j}$. Thus, we replace $x_{j}$ with $x_{j} e_{j}$ and assume that $x_{1}^{*}=x_{1}, x_{2}^{*}=x_{2}^{-1}$. Write $x_{1}=x_{1}^{m} x_{1}^{n}$, where $x_{1}^{m} \in P$ and $x_{1}^{n}$ is a $p^{\prime}$-element. Then each $a_{i}$ commutes with $x_{1}^{m}$. Thus, we replace $x_{1}$ with $x_{1}^{n}$ and assume that $x_{1}$ is a $p^{\prime}$-element, and similarly for $x_{2}$.

Suppose that $\left(a_{i}, x_{j}\right) \neq 1$. By minimality of $|G|$, we have $G=\left\langle a_{i}, x_{j}\right\rangle$. Let $x_{j}^{r}$ be a power of $x_{j}$ having prime order. Since $G$ is centreless, we see that $\left(a_{i}, x_{j}^{r}\right) \neq 1$. Thus, by minimality of $|G|$, we have $G=\left\langle a_{i}, x_{j}^{r}\right\rangle$. We therefore replace $x_{j}$ with $x_{j}^{r}$ and assume that $x_{j}$ has prime order $q$. By the same argument, we may assume that $o\left(a_{i}\right)=p$.

We have four cases to consider. But one of them is essentially done. Indeed, to prove that $\left(a_{2}, x_{2}\right)=1$, we look to that same case in [6]. Naturally, terms of the form $g+g^{*}$ must be replaced with $g-g^{*}$. This results in some sign changes, but the group elements remain the same, and we can follow the argument verbatim.

Next, let us show that $\left(a_{1}, x_{2}\right)=1$. Let $a=a_{1}, x=x_{2}, b=a^{x}, c=b^{x}$ and $d=c^{x}$. We have

$$
\begin{aligned}
0 & =\left[\left[a x-x^{-1} a, x-x^{-1}\right],\left[a^{2} x-x^{-1} a^{2}, x-x^{-1}\right]\right] \\
& =\left[a x^{2}+x^{-1} a x^{-1}-x a x-x^{-2} a, a^{2} x^{2}+x^{-1} a^{2} x^{-1}-x a^{2} x-x^{-2} a^{2}\right] .
\end{aligned}
$$

This expression consists of sums and differences of terms in $P x^{4}, P$ and $P x^{-4}$. As $x$ has odd order, these cosets are distinct. Thus, looking only at the terms in $P x^{4}$, we obtain

$$
a x^{2} a^{2} x^{2}+x a x^{2} a^{2} x+a^{2} x^{3} a x+x a^{2} x a x^{2}=a x^{3} a^{2} x+x a x a^{2} x^{2}+a^{2} x^{2} a x^{2}+x a^{2} x^{2} a x .
$$

Suppose, first of all, that each term on the left-hand side of our equation agrees with a term on the right-hand side. If $a x^{2} a^{2} x^{2}=a x^{3} a^{2} x$, then $\left(a^{2}, x\right)=1$, hence $(a, x)=$ 1 , as $a$ has odd order. If $a x^{2} a^{2} x^{2}=x a x a^{2} x^{2}$, then $(a, x)=1$. If $a x^{2} a^{2} x^{2}=a^{2} x^{2} a x^{2}$, then $\left(x^{2}, a\right)=1$, and again $(a, x)=1$. Thus, we can only have $a x^{2} a^{2} x^{2}=x a^{2} x^{2} a x$. Similarly, we can rule out all possibilities except for $x a x^{2} a^{2} x=a^{2} x^{2} a x^{2}$. But now note that $x a x^{2} a^{2} x=\left(a x^{2} a^{2} x^{2}\right)^{x}$. Therefore, $a^{2} x^{2} a x^{2}=\left(x a^{2} x^{2} a x\right)^{x}=x^{2} a^{2} x^{2} a$. That is, $\left(x^{2}, a^{2} x^{2} a\right)=1$, and hence $\left(x, a^{2} x^{2} a\right)=1$. But then $a x^{2} a^{2} x^{2}=x a^{2} x^{2} a x=a^{2} x^{2} a x^{2}$; that is, $\left(x^{2}, a\right)=1$, and once again we are done.

The only other possibility is that $p=3$ and three of the four terms on the left-hand side of our equation agree. There are several cases to consider here. If $b \in\langle a\rangle$, then $G=\langle a\rangle \rtimes\langle x\rangle$. But if this occurs, then the number of Sylow $q$-subgroups is $1+k q$ and divides 3 ; therefore, it is 1 , and $G$ is nilpotent. Thus, the product $\langle a\rangle \times\langle b\rangle$ is direct. If 
$c \in\langle a, b\rangle$, then $G=(\langle a\rangle \times\langle b\rangle) \rtimes\langle x\rangle$, and by a similar argument, $G$ is nilpotent. Thus, the product $\langle a\rangle \times\langle b\rangle \times\langle c\rangle$ is direct.

Now the terms on the left-hand side of our above equation are $a c^{2} x^{4}, b d^{2} x^{4}, a^{2} d x^{4}$ and $b^{2} c x^{4}$. As three of these agree, we must have either $a c^{2}=a^{2} d$ or $b d^{2}=b^{2} c$. In the first case, $a^{x^{3}}=d=a^{-1} c^{-1}$, and a straightforward calculation reveals that $a^{x^{8}}=a$. But also $a^{x^{q}}=a$, and therefore $(a, x)=1$. In the second case, we have a subgroup $(\langle b\rangle \times\langle c\rangle) \rtimes\langle x\rangle$ and, as we have noted above, such a group is abelian. Thus, $(b, x)=1$, and therefore $(a, x)=1$. This case is complete.

We have proved that $x_{2}$ is central. Since $G$ is centreless, * acts trivially upon $G / P$.

Next, let us show that $\left(a_{2}, x_{1}\right)=1$. Let $a=a_{2}, x=x_{1}, b=a^{x}$ and $c=b^{x}$. First of all, suppose that $b \in\langle a\rangle$. Then $G=\langle a\rangle \rtimes\langle x\rangle$, and $P=\langle a\rangle=P_{2}$. In particular, $\left(a^{x}\right)^{*}=\left(a^{x}\right)^{-1}$ as well. That is, $x^{-1} a^{-1} x=x a^{-1} x^{-1}$, hence $\left(x^{2}, a\right)=1$, and therefore $(a, x)=1$.

For our second case, assume that the product $\langle a\rangle \times\langle b\rangle$ is direct, but $c \in\langle a, b\rangle$. Then $G=(\langle a\rangle \times\langle b\rangle) \rtimes\langle x\rangle$. As we have noted above, if $p=3$, then $G$ is abelian, so assume that $p>3$. We have

$$
\begin{aligned}
0 & =\left[\left[a-a^{-1}, a x-x a^{-1}\right],\left[a-a^{-1}, x a-a^{-1} x\right]\right] \\
& =\left[a^{2} x+a^{-1} x a^{-1}-a x a-x a^{-2}, a x a+a^{-2} x-x a^{2}-a^{-1} x a^{-1}\right] .
\end{aligned}
$$

That is,

$$
\begin{aligned}
& a^{2} x a x a+a^{2} x a^{-2} x+a^{-1} x a^{-3} x+a x a x a^{2}+x a^{-2} x a^{2}+x a^{-3} x a^{-1} \\
+ & \operatorname{axaxa}^{-2}+a^{-2} x a x a+a^{-2} x^{2} a^{-2}+x a^{4} x+x a x a^{-1}+a^{-1} x a x \\
= & a^{2} x^{2} a^{2}+a^{2} x a^{-1} x a^{-1}+a^{-1} x a^{-1} x a^{2}+a x a^{-1} x+x a^{-1} x a+x a^{-4} x \\
+ & a x a^{3} x++a^{-2} x a^{2} x+a^{-2} x a^{-1} x a^{-1}+x a^{3} x a+x a^{2} x a^{-2}+a^{-1} x a^{-1} x a^{-2} .
\end{aligned}
$$

Suppose, first of all, that every term on the left-hand side of our equation agrees with a term on the right-hand side. Let us consider the possibilities for $a^{2} x a^{-2} x$. If $a^{2} x a^{-2} x=a^{2} x^{2} a^{2}$, then $a^{-2} x=x a^{2}$, hence $\left(a^{2}\right)^{x}=a^{-2}$, and therefore $\left(a^{2}, x^{2}\right)=1$. That is, $(a, x)=1$. Similarly, if $a^{2} x a^{-2} x \in\left\{a^{2} x a^{-1} x a^{-1}, a x a^{-1} x, x a^{-4} x, a^{-2} x a^{2} x\right\}$, then $(a, x)=1$. If $a^{2} x a^{-2} x=a x a^{3} x$, then $a x a^{-2}=x a^{3}$, so $a^{x^{-1}}=a^{5}$. As $x$ has finite order, we have $a^{x} \in\langle a\rangle$ as well, which is not permitted. If $a^{2} x a^{-2} x=x a^{2} x a^{-2}$, then $a^{2} b^{-2} x^{2}=$ $b^{2} c^{-2} x^{2}$. Thus, $c^{2}=a^{-2} b^{4}$. As our group has odd order, $c=a^{-1} b^{2}$. But this means that $a^{x}=b$ and $a^{x^{2}}=a^{-1} b^{2}$. An easy induction reveals that for all positive integers $i$, $a^{x^{i}}=a^{1-i} b^{i}$. In particular, $a^{x^{p}}=a$. But $a^{x^{q}}=a$ as well, and therefore $(a, x)=1$.

We are left with five possibilities for $a^{2} x a^{-2} x$. If, for instance, $a^{2} x a^{-2} x=$ $a^{-1} x a^{-1} x a^{2}$, then $a^{2} b^{-2} x^{2}=a^{-1} b^{-1} c^{2} x^{2}$, hence $c^{2}=a^{3} b^{-1}$. Considering each of the five possibilities in turn, and isolating a power of $c$, we obtain

$$
a^{2} x a^{-2} x= \begin{cases}x a^{-1} x a & \Longrightarrow c=a^{2} b^{-1} \\ a^{-1} x a^{-1} x a^{-2} & \Longrightarrow c^{2}=a^{-3} b \\ a^{-1} x a^{-1} x a^{2} & \Longrightarrow c^{2}=a^{3} b^{-1} \\ a^{-2} x a^{-1} x a^{-1} & \Longrightarrow c=a^{-4} b \\ x a^{3} x a & \Longrightarrow c=a^{2} b^{-5}\end{cases}
$$

On the other hand, let us consider the possibilities for $x a^{-2} x a^{2}$. Reasoning as above, we see that if $x a^{-2} x a^{2} \in\left\{a^{2} x^{2} a^{2}, a^{-1} x a^{-1} x a^{2}, x a^{-1} x a, x a^{-4} x, x a^{2} x a^{-2}\right\}$, then 
$(a, x)=1$. If $x a^{-2} x a^{2}=a^{-2} x a^{2} x$, then $b^{-2} c^{2} x^{2}=a^{-2} b^{2} x^{2}$, hence $c^{2}=a^{-2} b^{4}$. As above, we find that $(a, x)=1$. If $x a^{-2} x a^{2}=x a^{3} x a$, then $a^{-2} x a=a^{3} x$, hence $a^{x}=a^{5} \in\langle a\rangle$, which is not allowed.

We are left with five possibilities for $x a^{-2} x a^{2}$, namely

$$
x a^{-2} x a^{2}=\left\{\begin{array}{ll}
a^{2} x a^{-1} x a^{-1} & \Longrightarrow c^{3}=a^{2} b \\
a^{-1} x a^{-1} x a^{-2} & \Longrightarrow c^{4}=a^{-1} b \\
a x a^{3} x & \Longrightarrow c^{2}=a b^{5} \\
a x a^{-1} x & \Longrightarrow c^{2}=a b \\
a^{-2} x a^{-1} x a^{-1} & \Longrightarrow c^{3}=a^{-2} b
\end{array} .\right.
$$

Now we have 25 cases to consider, given by the five possibilities for $a^{2} x a^{-2} x$ and five for $x a^{-2} x a^{2}$. For instance, if $c^{2}=a^{-3} b$ and $c^{3}=a^{2} b$, then $a^{-9} b^{3}=c^{6}=a^{4} b^{2}$. That is, $a^{13} b^{-1}=1$. Making use of the assumptions that $p>3,\langle a\rangle \times\langle b\rangle$ is a direct product, and $c=a^{-1} b^{2}$ is not permitted, calculations of the above type yield a contradiction in every case. (It is, perhaps, worth mentioning that in the situation where $c^{2}=a^{3} b^{-1}$ and $c^{3}=a^{2} b$, we obtain $c=\left(a^{2} b\right)\left(a^{3} b^{-1}\right)^{-1}=a^{-1} b^{2}$, which is not allowed.)

Thus, it is impossible for every term on the left-hand side of our equation to agree with a term on the right-hand side. As there are only 12 terms on each side, we are done if $p \geq 13$. Suppose that $p=11$. If 11 terms on the left-hand side agree, then at least two of $a^{2} x a^{-2} x, x a^{4} x$ and $a^{-1} x a^{-3} x$ must agree. But these are, respectively, equal to $a^{2} b^{-2} x^{2}, b^{4} x^{2}$ and $a^{-1} b^{-3} x^{2}$. As the product $\langle a\rangle \times\langle b\rangle$ is direct, these elements are pairwise distinct. Thus, the case $p=11$ is done as well.

As we have ruled out the case $p=3$ earlier, we may assume that $p \in\{5,7\}$. Now $G=(\langle a\rangle \times\langle b\rangle) \rtimes\langle x\rangle$. The number of Sylow $q$-subgroups is $1+k q$ and divides $p^{2}$. As we are assuming that $k \neq 0$, we can only have $q=3$. Let $c=a^{i} b^{j}$. Then as $c^{x}=a^{x^{3}}=a$, we have $a=b^{i}\left(a^{i} b^{j}\right)^{j}=a^{i j} b^{i+j^{2}}$. In particular, $i j \equiv 1(\bmod p)$ and $i+j^{2} \equiv 0(\bmod p)$. Thus, we see that $-j^{3} \equiv 1(\bmod p)$. If $p=5$, the only solution is $j=i=-1$. If $p=7$, we have three possibilities: $j=i=-1 ; j=5, i=3$ and $j=3, i=5$.

With this in mind, let us calculate the terms on the left-hand side of our equation, multiplying on the right by $x^{-2}$ for convenience. Assuming that $i=j=-1$, we get

$$
a, a^{2} b^{-2}, a^{-1} b^{-3}, a^{-1} b^{-1}, a^{-2} b^{-4}, a b^{-2}, a^{3} b^{3}, a^{-3}, b^{2}, b^{4}, a b^{2} \text { and } a^{-1} b .
$$

We immediately see that there is no possibility of five or more terms agreeing.

Next, let $p=7, i=3, j=5$. Then we have

$$
a^{5} b^{6}, a^{2} b^{5}, a^{6} b^{4}, b^{4}, a^{6} b, a^{4} b^{6}, a^{2} b^{5}, a b^{6}, a^{6} b^{4}, b^{4}, a^{4} b^{3} \text { and } a^{6} b .
$$

Again, we cannot have seven terms agreeing.

Finally, let $p=7, i=5, j=3$. Then we get

$$
b^{4}, a^{2} b^{5}, a^{6} b^{4}, a^{4}, a^{3} b^{4}, a^{2} b, a^{5} b^{2}, a^{3} b^{4}, a^{2} b, b^{4}, a^{2} b^{5} \text { and } a^{6} b .
$$

Once again, seven terms cannot agree, and this case is complete.

Let us assume, therefore, that the product $\langle a\rangle \times\langle b\rangle \times\langle c\rangle$ is direct. Then

$$
\begin{aligned}
0 & =\left[\left[a-a^{-1}, a x-x a^{-1}\right],\left[a^{2}-a^{-2}, a^{2} x-x a^{-2}\right]\right] \\
& =\left[\left(a^{2}+a^{-1} b^{-1}-a b-b^{-2}\right) x,\left(a^{4}+a^{-2} b^{-2}-a^{2} b^{2}-b^{-4}\right) x\right] .
\end{aligned}
$$


Expanding and multiplying on the right by $x^{-2}$, we see that the following expression is equal to zero, namely

$$
\begin{aligned}
& a^{2} b^{4}+a^{2} b^{-2} c^{-2}+a^{-1} b^{3}+a^{-1} b^{-3} c^{-2}+a b^{3} c^{2}+a b c^{-4}+c^{2}+b^{-2} c^{-4} \\
+ & a^{4} b c+a^{4} c^{-2}+a^{-2} b^{-1} c+a^{-2} b^{-2} c^{-2}+a^{2} b^{4}+a^{2} b c^{-1}+b^{-2}+b^{-5} c^{-1} \\
- & a^{2} b^{2} c^{2}-a^{2} c^{-4}-a^{-1} b c^{2}-a^{-1} b^{-1} c^{-4}-a b^{5}-a b^{-1} c^{-2}-b^{2}-b^{-4} c^{-2} \\
- & a^{4} b^{2}-a^{4} b^{-1} c^{-1}-a^{-2}-a^{-2} b^{-3} c^{-1}-a^{2} b^{3} c-a^{2} b^{2} c^{-2}-b^{-3} c-b^{-4} c^{-2} .
\end{aligned}
$$

Since the product is direct, we observe that $b^{2}$ cannot cancel with any other term. This case is complete, and we conclude that $\left(a_{2}, x_{1}\right)=1$.

We conclude that $P_{2}$ is central and so, as $G$ is centreless, $P=P_{1}$.

Finally, we show that $\left(a_{1}, x_{1}\right)=1$. Let $a=a_{1}$ and $x=x_{1}$. But since $P=P_{1}$, we have $\left(a^{x}\right)^{*}=a^{x}$. That is, $x^{-1} a x=x a x^{-1}$. Thus, $\left(x^{2}, a\right)=1$. Therefore, $(x, a)=1$, and the proof is complete.

We can now use Theorem 1 to prove our main result.

Proof of Theorem 2. Suppose that $(F G)^{-}$is Lie metabelian, where $G$ is not abelian. We know that $(F G)^{-}$satisfies a polynomial identity. Hence, in view of Lemmas 7 and $8, G$ is locally finite. If $g$ and $h$ are non-commuting elements of $G$, then it suffices to assume that $G=\left\langle g, h, g^{*}, h^{*}\right\rangle$. That is, we may take $G$ to be finite. By Theorem $1, G$ is nilpotent. If $p=0$, then by Lemma 1 , we are done, so let $p$ be greater than 3 . Then $G$ is the direct product of a $p$-group and a $p^{\prime}$-group. By Lemma 1 , the $p^{\prime}$-group is abelian. We may, therefore, assume that $G$ is a $p$-group.

Now, since $\left[(F G)^{+},(F G)^{+}\right] \subseteq(F G)^{-}$, we see that $(F G)^{+}$has Lie derived length at most 3. By Lemma 4, $p \leq 7$, and the second part of the theorem is proved. Therefore, let $F G$ have the classical involution, and suppose that $G$ is a counterexample of the smallest order. If $G^{\prime}$ is not central, then since $\left(G^{\prime}, G\right) \subsetneq G^{\prime}$, we find that $G /\left(G^{\prime}, G\right)$ gives a counterexample of smaller order. Therefore, $G^{\prime}$ is central. Also, if $\left|G^{\prime}\right|>p$, then taking an element $z$ of order $p$ in $G^{\prime}$, we note that $G /\langle z\rangle$ gives a counterexample of smaller order. Therefore, $\left|G^{\prime}\right|=p$. Lemma 3 now completes the proof.

Levin and Rosenberger in [13] showed that when $p=3, F G$ is Lie metabelian if and only if $G$ is nilpotent and $\left|G^{\prime}\right|=1$ or 3 . Thus, we surely cannot allow $p=3$ in Theorem 2 . Furthermore, we cannot extend Theorem 2 to an arbitrary involution when $p \in\{5,7\}$, as the following proposition demonstrates. As a straightforward consequence, we see that Lemma 3 does not extend to group rings endowed with the linear extension of an arbitrary group involution.

For any group $G$, write $\Delta(G)$ for the augmentation ideal; that is, the set of all elements of $F G$ whose coefficients sum to zero.

Proposition. Let $F$ be a field of characteristic $p \in\{5,7\}$. Then there exists a non-abelian finite p-group $G$ with involution * such that under the induced involution, $(F G)^{-}$is Lie metabelian.

Proof. Suppose that $G$ is a finite $p$-group with $G^{\prime}=\langle z\rangle$ of order $p$. Let $*$ be trivial on $G / G^{\prime}$. Then for any $a, b, c, d \in G$, we note that $a-a^{*}=a-a z^{i}$, for some $i$, so this is $a\left(1-z^{i}\right)=a \delta_{1}$, with $\delta_{1} \in \Delta\left(G^{\prime}\right)$ (which is central in $F G$ ), and similarly for $b, c, d$. 
That is,

$$
\left[\left[a-a^{*}, b-b^{*}\right],\left[c-c^{*}, d-d^{*}\right]\right]=\delta_{1} \delta_{2} \delta_{3} \delta_{4}[[a, b],[c, d]],
$$

for some $\delta_{j} \in \Delta\left(G^{\prime}\right)$. But now $[a, b]=a b(1-(b, a))=a b \delta_{5}$ for some $\delta_{5} \in \Delta\left(G^{\prime}\right)$. Thus, we have

$$
\left[\left[a-a^{*}, b-b^{*}\right],\left[c-c^{*}, d-d^{*}\right]\right]=\delta_{1} \delta_{2} \delta_{3} \delta_{4} \delta_{5} \delta_{6}[a b, c d],
$$

and similarly, this is

\section{$\delta_{1} \delta_{2} \delta_{3} \delta_{4} \delta_{5} \delta_{6} \delta_{7} a b c d$}

for some $\delta_{j} \in \Delta\left(G^{\prime}\right)$. However, it is easy to verify (see, for instance, the proof of [10, Lemma 1.1.1]) that $\Delta\left(G^{\prime}\right)^{p}=0$. Thus, $(F G)^{-}$is Lie metabelian.

It remains to check that there exists a non-abelian finite $p$-group $G$ such that $G^{\prime}$ has order $p$, and $G$ has an involution $*$ acting trivially upon $G / G^{\prime}$. But let $G$ be the nonabelian group of order $p^{3}$ having exponent $p$ (the Heisenberg group). Choosing noncommuting elements $a$ and $b$, and writing $z=(b, a)$, we know that the elements of $G$ are uniquely written as $a^{i} b^{j} z^{k}$, with $0 \leq i, j, k<p$. Define a map $*$ via $\left(a^{i} b^{j} z^{k}\right)^{*}=a^{i} b^{j} z^{i j-k}$. We can see that

$$
\left(\left(a^{i} b^{j} z^{k}\right)^{*}\right)^{*}=a^{i} b^{j} z^{i j-(i j-k)}=a^{i} b^{j} z^{k}
$$

as required, and

$$
\left(a^{i} b^{j} z^{k} a^{l} b^{m} z^{n}\right)^{*}=\left(a^{i+l} b^{j+m} z^{k+n+j l}\right)^{*}=a^{i+l} b^{j+m} z^{(i+l)(j+m)-(k+n+j l)},
$$

whereas

$$
\left(a^{l} b^{m} z^{n}\right)^{*}\left(a^{i} b^{j} z^{k}\right)^{*}=a^{l} b^{m} z^{l m-n} a^{i} b^{j} z^{i j-k}=a^{i+l} b^{j+m} z^{i m+l m-n+\ddot{j}-k},
$$

and these are equal. Thus, $*$ is an involution on $G$. As it is clearly trivial on $G / G^{\prime}$, we are done.

\section{REFERENCES}

1. S. A. Amitsur, Rings with involution, Israel J. Math. 6 (1968), 99-106.

2. Zs. Balogh, Lie derived length and involutions in group algebras, J. Pure Appl. Algebra 216 (2012), 1282-1287.

3. Zs. Balogh and T. Juhász, Derived lengths of symmetric and skew symmetric elements in group algebras, JP J. Algebra Number Theory Appl. 12 (2008), 191-203.

4. O. Broche Cristo, E. Jespers, C. Polcino Milies and M. Ruiz Marín, Antisymmetric elements in group rings II, J. Algebra Appl. 8 (2009), 115-127.

5. F. Catino, G. T. Lee and E. Spinelli, The bounded Lie Engel property on torsion group algebras, J. Pure Appl. Algebra 215 (2011), 2639-2644.

6. F. Catino, G. T. Lee and E. Spinelli, Group algebras whose symmetric elements are Lie metabelian, Forum Math. (to appear). doi:10.1515/forum-2012-0005.

7. A. Giambruno, C. Polcino Milies and S. K. Sehgal, Group algebras of torsion groups and Lie nilpotence, J. Group Theory 13 (2010), 221-231.

8. A. Giambruno and S. K. Sehgal, Lie nilpotence of group rings, Comm. Algebra 21 (1993), 4253-4261.

9. G. T. Lee, The Lie $n$-Engel property in group rings, Comm. Algebra 28 (2000), 867-881. 
10. G. T. Lee, Group identities on units and symmetric units of group rings (Springer, London, 2010).

11. G. T. Lee, S. K. Sehgal and E. Spinelli, Group algebras whose symmetric and skew elements are Lie solvable, Forum Math. 21 (2009), 661-671.

12. G. T. Lee and E. Spinelli, Group rings whose symmetric units are solvable, Comm. Algebra 37 (2009), 1604-1618.

13. F. Levin and G. Rosenberger, Lie metabelian group rings, in Group and semigroup rings, North-Holland Mathematical Studies 126 (Elsevier, Amsterdam, Netherlands, 1986), 153-161.

14. F. Levin and G. Rosenberger, On Lie metabelian group rings, Results Math. 26 (1994), $83-88$.

15. D. S. Passman, The algebraic structure of group rings (Wiley, New York, 1977).

16. D. J. S. Robinson, A course in the theory of groups, 2nd ed. (Springer, New York, 1996).

17. S. Siciliano, On the Lie algebra of skew-symmetric elements of an enveloping algebra, J. Pure Appl. Algebra 215 (2011), 72-76. 